\title{
Algebras which are independently generated by every $n$ elements
}

\author{
by

\section{S. Swierczkowski (Wroctaw)}

\section{Preliminaries and results}

By an algebra si we mean a pair $(A, F)$ where $A$ is a set and $\boldsymbol{F}$ is a family of functions of finitely many variables defined on $A$ and $A$-valued. $F$ is called the class of fundamental operations. The olass of algebraic operations is, by definition, the class of operations $\boldsymbol{A}$ generated by $\boldsymbol{F}$, i. e. the smallest class $\boldsymbol{A}$ such that $\boldsymbol{A}$ contains $\boldsymbol{F}$, all identity operations belong to $A$ and $A$ is closed with respect to composition. The subclass of all algebraic operations of $n$ variables will be denoted by $\boldsymbol{A}^{(n)}$. The above definitions are given in a more detailed form in [3]; we use here the same notation.

Following E. Marczewski [3] we say that $N \subset A$ is a set of independent elements if, for each sequence of $n$ different elements $a_{1}, \ldots, a_{n} \in N$ and for each pair of operations $f, g \in \boldsymbol{A}^{(n)}$, the equality

$$
f\left(a_{1}, \ldots, a_{n}\right)=\mathfrak{\jmath}\left(a_{1}, \ldots, a_{n}\right)
$$

implies that $f$ and $g$ are identical in $s$.

We shall call the identity operations also trivial operations. More exactly: An operation $f\left(x_{1}, \ldots, x_{k}\right)$ is called trivial if, for a certain $l \leqslant k$, we have $f\left(x_{1}, \ldots, x_{k}\right)=x_{l}$ for all values of $x_{1}, \ldots, x_{k}$. If all algebraic operations are trivial then the algebra will be called trivial. For $A=\left\{a_{1}, \ldots, a_{n}\right\}$ and $\boldsymbol{F}=\{f\}$ we shall. write $\left(a_{1}, \ldots, a_{n} ; f\right)$ instead of $(A, F)$. Two algebras, $\left(A, F_{1}\right)$ and $\left(A, F_{2}\right)$, having the same class of all algebraic operations will be treated here as identical.

We say that a set $B C A$ generates $s t$ if each $x \in A$ is the result of an algebraic operation applied to some elements in $B$. Let $\bar{S}$ denote the cardinal of the set $S$. We then say that the algebra is independently generated by every $n$ elements if each set $B C A$ satisfying $\bar{B}=n$ is a set of independent elements and $B$ generates $s t$. In this paper we show some properties of those algebras. The results were announced in paper [4]. 
THEOREM 1. If all elements of an algebra $q$ are independent and. $\bar{A} \neq 2$, then $s$ is a trivial algebra. There exists a non-trivial two-element algebra on all elements of which are independent.

The algebra $M$ has been found by $\mathrm{E}$. Marczewsiki. It is evident that all elements of a trivial algebra are independent. Hence, if the trivial algebra has $n$ elements, we obtain an example of an algebra which is independently generated by every $n$ elements. If $n>3$, then there are no other algebras of this kind since we have

THEOREM 2. Let $n>3$. If . is an algebra such that $\overline{\bar{A}} \geqslant n$ and st is independently generated by every $n$ elements, then $\alpha$ is the trivial alyebra with $n$ elements.

The assumption $n>3$ is essential in this theorem. For $n=3$ we consider the following:

Let us put $s l_{0}=\left(a, b, e, d ; f_{0}\right)$ where $f_{0}=f_{0}(x, y, z)$ is the operation which associates with every three distinct elements of the set $\{a, b, c, d\}$ the remaining one and satisfies identically

$$
f_{0}(x, x, y)=f_{0}(x, y, x)=f_{0}(y, x, x)=y \text {. }
$$

THEOREM 3. The algebra $s \mathrm{~L}_{0}$ is independently generated by every three elemeints.

THEOREM 4. silo is the unique algebra which is non-trivial, has at least three elements and is independently generated by every three elements.

There exist non-trivial algebras which are independently generated by every two elements. An example is the algebra $M$ considered in Theorem 1. Another kind of example gives the following theorem, which was communicated to me by A. M. Macbeath.

THEOREM 5. Let $K$ be a field, let $A$ be the set of all elements of $K$ and let $F$ be the elass of all operations $f\left(x_{1}, x_{2}\right)=\lambda x_{1}+(1-\lambda) x_{2} ; \lambda \in K$. Then the algebra $\mathcal{A}=(A, F)$ is independently generated by every two elements.

It follows from this theorem that there is, for every number $p^{k}$ ( $p$ prime, $k$ natural), an algebra with $p^{k}$ elements which is independently generated by every two elements. Also the converse of this result is true:

THEOREM 6. If $s$ is a finite algebra which is independently generated by every two elements, then $\overline{\bar{A}}$ is a power of a prime.

In view of Theorems 5 and 6 (cf. also [5]) one might suspect that each algebra which is independently generated by every two elements is defined, as in Theorem 5, by a corresponding field. This, however, is not true and the simplest counter-example is the algebra $m$ of Theorem 1.

For every set $\boldsymbol{A}$ there is a class of operations $\boldsymbol{F}$ such that $g l=(A, \boldsymbol{F})$ is an algebra which is independently generated by every element. To prove this we introduce in $A$ a group addition so that $A$ is an Abelian group. We associate with every $a \in A$ the operation $f_{u}(x)=a+x$ and we denote by $\boldsymbol{F}$ the class of all those operations. Then $c t$ is generated by every element. Moreover, every element is independent since $f_{a}$ are the only operations of one variable.

\section{Algebras with all elements independent}

In this section we shall prove Theorem 1 . Denote by $n$ the cardinal of $A$. The theorem is trivial for $n=1$. We assume therefore that $n \geqslant 3$ and we have to show that every algebraic operation $f\left(x_{1}, \ldots, x_{k}\right)$ is a trivial one. We consider the possibilities: $k<n$ and $k \geqslant n$ (the latter obviously only for finite $n$ ).

For $k<n$ it is convenient, for a further application, to derive the result from the weaker assumption $\overline{\bar{A}} \geqslant n$. So we first prove the following:

(a) If $\overline{\bar{A}} \geqslant n$ and every $n$ elements of $s$ are independent, then $A^{(k)}$ contains, for each $k<n$, only trivial operations.

Proof of $(\alpha)$. Let $k<n$ and let $f \in A^{(k)}$. Consider $k$ different elements $a_{1}, \ldots, a_{k} \in A$. The elements $a_{1}, \ldots, a_{k}, a_{k+1}=f\left(a_{1}, \ldots, a_{k}\right)$ are obviously not independent. Thus they cannot be different, by $k+1 \leqslant n$, and we have

$$
f\left(a_{1}, \ldots, a_{k}\right)=a_{l} \quad \text { for some } \quad l<k .
$$

From the independence of the $a_{i}$ it follows that we have identically $f\left(x_{1}, \ldots, x_{k}\right)=x_{l}$, so that $f$ is a trivial operation. Hence $(\alpha)$ is proved

Now let $k \geqslant n, f \in A^{(k)}$ and let $a_{1}, \ldots, a_{k} \in A$. Suppose that the element $a_{i+1}=f\left(a_{1}, \ldots, a_{k}\right)$ is different from all $a_{i}, i \leqslant k$. Then, if $r$ is the number of distinct elements $a_{i_{1}}, \ldots, a_{i_{r}}$ occuring in the sequence $\left\langle a_{1}, \ldots, a_{k}\right\rangle$, we infer that all $a_{i_{1}}, \ldots, a_{i_{r}}, a_{k+1}$ are different and thus independent. This is impossible since $a_{k+1}$ is the result of an algebraic operation on $a_{i_{1}}, \ldots, a_{i_{r}}$. So

$$
f\left(a_{1}, \ldots, a_{k}\right)=a_{n}
$$

for some $u \leqslant k$. Let $S=\{1, \ldots, k\}$ and let $A\left(a_{1}, \ldots, a_{k}\right)$ be the subset of all $u \in S$ satisfying $(*)$. We shall show that $f$ is a trivial operation if we prove that there is a number $u$ such that $(*)$ holds for that $u$ and for arbitrary $a_{1}, \ldots, a_{k} \in A$. Equivalently, we have to show that the intersection of all sets $A\left(a_{1}, \ldots, a_{k}\right)$ is non-empty.

Suppose we have a one-to-one mapping of $A$ onto itself. Let $a^{\prime}$ denote the image of $a$. It follows from the independence of all elements of $s t$ (cf. [3], sec. 2 (ii)) that (*) holds with the same numbers $u$ for $a_{1}, \ldots, a_{k}$ and $a_{1}^{\prime}, \ldots, a_{k}^{\prime}$. Thus $A\left(a_{1}, \ldots, a_{k}\right)$ depends only on the decomposition $\delta$ of the set of indices $S$ in the disjoint sets $D_{1}, \ldots, D_{m}$ containing indices of equal $a_{i}$ (so that $a_{i}=a_{j}$ if and only if $i, j$ belong to the same $D_{l}$ 
whence $m \leqslant n=\vec{A}$. Evidently $\Delta\left(a_{1}, \ldots, a_{k}\right)$ is one of those sets $D_{l}$ and since it depends only on the decomposition $\delta$, it will be denoted by $p \delta$. We observe that every decomposition of $S$ into not more than $n$ subsets is realized by some sequence $\left\langle a_{1}, \ldots, a_{k}\right\rangle$.

DeFinition. For any decompositions $\delta, \delta^{\prime}$ we write $\delta<\delta^{\prime}$ if, for each set $D_{i}$ of $\delta$, there is a set $D_{j}^{\prime}$ of $\delta^{\prime}$ which contains $D_{i}$.

Let us prove that $\delta<\delta^{\prime}$ implies $q \delta \subset \varphi \delta^{\prime}$. Indeed, if $\delta<\delta^{\prime}$ and $a_{1}, \ldots, a_{k}$ is a sequence that determines the decomposition $\delta$, then there is a mapping of $A$ into itself such that the sequence $\left\langle a_{1}^{\prime}, \ldots, a_{k}^{\prime}\right\rangle$, which is the image of $\left\langle a_{1}, \ldots, a_{k}\right\rangle$, determines the decomposition $\delta^{\prime}$. From the independence of the elements of $l$ it follows ([3], see. 2, (ii)) that if $(*)$ holds for some $u$ and $a_{1}, \ldots, a_{k}$, then it holds for the same $u$ for $a_{1}^{\prime}, \ldots, a_{h}^{\prime}$. Thus $q \delta C_{\varphi} \delta^{\prime}$.

We have to prove that the intersection of all po is unn-empty. This follows from the lemma

(B) If we have a fixed number $n \geqslant 3, S$ is a finite set, and to enery decomposition $\delta$ of $S$ in not more than $n$ disjoint subsets corresponds $a$ set $\varphi \delta$ of that decomposition so that $\delta<\delta^{\prime}$ implies $m \delta C \propto \delta^{\prime}$, then the intersection of all po is non-empty.

Pro of of $(\beta)$. The assumption $n \geqslant 3$ means that the correspoudence $\delta \rightarrow \infty \delta$ is defined for all decompositions in not more than three subsets.

It is sufficient to verify that, for any $\delta^{\prime}, \delta^{\prime \prime}$ the set $q \delta^{\prime} \cap \varphi \delta^{\prime \prime}$ is also a $p \delta$. We prove first that $\varphi \delta^{\prime}$ and $p \delta^{\prime \prime}$ are not disjoint. Consider the decompositions $\delta_{1}, \delta_{3}$

$$
\$=\phi \delta^{\prime} \cup B, \quad S=\varphi \delta^{\prime \prime} \cup C,
$$

where $B$ and $C$ are uniquely determined. We have $\delta^{\prime}<\delta_{1}, \delta^{\prime \prime}<\delta_{2}$ and this implies $\varphi \delta_{1}=\varphi \delta^{\prime}, \varphi \delta_{2}=q \delta^{\prime \prime}$. Suppose that $\varphi \delta^{\prime}$ and $q \delta^{\prime \prime}$ are disjoint. Then the decomposition $\delta^{*}$

$$
B=q \delta^{\prime} \cup \varphi \delta^{\prime \prime} \cup D
$$

satisfies $\delta^{*}<\delta_{1}, \delta^{*}<\delta_{2} . q \delta^{*}$ is contained in both $q \delta_{1}$ and $q \delta_{2}$, which contradiets $\varphi \delta_{1} \cap \varphi \delta_{2}=\varphi \delta^{\prime} \cap \varphi \delta^{\prime \prime}=\emptyset$ (Ø is the empty set).

Now consider the decomposition $\delta$ defined by

$$
S=\left(\varphi \delta^{\prime} \cap \varphi \delta^{\prime \prime}\right) \cup\left(\varphi \delta^{\prime}-\varphi \delta^{\prime \prime}\right) \cup B .
$$

From $\delta<\delta_{1}$ follows $\varphi \delta \subset \varphi \delta_{1}=\varphi \delta^{\prime}$ and thus $\varphi \delta \neq B$. Since two sets $\varphi \delta, \varphi \delta^{\prime \prime}$ are never disjoint, we have $\varphi \delta=\varphi \delta^{\prime} \cap \varphi \delta^{\prime \prime}$. This completes the proof of $(\beta)$ and of the first part of Theorem 1 .

Let $\eta f=(a, b ; f)$ where $f=f(x, y, z)$ is the operation defined on $\{a, b\}$ so that identically

$$
f(x, x, y)=f(x, y, x)=f(y, x, x)=x .
$$

Hence $f$ is non-trivial and thus the algebra $c h$ is non-trivial. All elements of $T h$ are independent, since, for any algebraic operations $g, h$ of two variables, the equality $g(a, b)=h(a, b)$ implies $g(b, a)=h(b, a)$ because of symmetry and $g(x, x)=x=h(x, x)$ holds since there is no non-trivial operation of one variable in $M$.

\section{Algebras which have more than three independent generators}

We shall now prove Theorem 2. The result follows by Theorem 1 if we verify that there are only $n$ elements in $A$. If $n$ is infinite, then, by ( $\alpha$ ), every operation is trivial and hence $A$ contains only the $n$ generators. If $n$ is finite, then it is enough to prove that $A^{(n)}$ contains only trivial operations.

Let us suppose that there is in $\boldsymbol{A}^{(n)}$ a non-trivial operation $f\left(x_{1}, \ldots, x_{n}\right)$. By $(\alpha)$, the operation on $n-1$ variables $f\left(x_{1}, x_{2}, x_{2}, x_{4}, \ldots, x_{n}\right)$ is trivial, whence it is identically equal to one of the variables $x_{1}, x_{2}, x_{4}, \ldots, x_{n}$. Certainly one of the two variables $x_{1}, x_{4}$ (we have $n \geqslant 4$ ) is not identically equal to $f\left(x_{1}, x_{2}, x_{2}, x_{4}, \ldots, x_{n}\right)$ and we may assume that it is the variable $x_{1}$, performing in the opposite case a suitable rearrangement of indices. Therefore, identically

$$
f\left(x_{1}, x_{2}, x_{2}, x_{4}, \ldots, x_{n}\right)=x_{2} \text { or } x_{4} \text { or } \ldots \text { or } x_{n} .
$$

Let $a_{1}, \ldots, a_{n} \in A$ be distinct elements and let $a_{n+1}=f\left(a_{1}, \ldots, a_{n}\right)$. Since $f$ is non-trivial and $a_{1}, \ldots, a_{n}$ are independent, we have $a_{n+1}$ $\neq a_{1}, \ldots, a_{n}$. The elements $a_{2}, \ldots, a_{n+1}$, being different, generate the algebra. Hence, for a certain algebraic operation $h, a_{1}=h\left(a_{2}, \ldots, a_{n+1}\right)$, i. e.

$$
a_{1}=h\left(a_{2}, a_{3}, a_{4}, \ldots, f\left(a_{1}, a_{2}, a_{3}, \ldots, a_{n}\right)\right) .
$$

Since all elements appearing in the above equality are independent, the equality holds identically, e. g. holds also if we put $a_{2}$ in place of $a_{3}$. Now $f\left(a_{1}, a_{2}, a_{2}, a_{4}, \ldots, a_{n}\right)$ is one of the elements $a_{2}, a_{4}, \ldots, a_{n}$ and so $a_{1}$ is the result of an algebraic operation on $a_{2}, a_{4}, \ldots, a_{n}$ in spite of the independence of $a_{1}, \ldots, a_{n}$. We have obtained a contradiction and we have proved the theorem.

\section{The algebra sto}

Fundamental properties. Let us prove Theorem 3. Since evidently $q_{0}$ is generated by every three elements, we have to show that every three elements are independent. Let us denote by $e_{1}, e_{2}, e_{3}$ the three trivial operations in $\boldsymbol{A}_{0}^{(3)}$ so that identically

$$
e_{1}(x, y, z)=x, \quad e_{2}(x, y, z)=y, \quad e_{3}(x, y, z)=z
$$

Fundamenta Mathematicae, T. XLIX. 
It is easy to check that the class of operations $\Phi=\left\{t_{0}, e_{1}, e_{2}, e_{3}\right\}$ has the following property:

If $h_{1}, h_{2}, h_{3} \in \Phi$, then the operation $h$ defined by the formula

$$
h(x, y, z)=f_{0}\left(h_{1}(x, y, z), h_{2}(x, y, z), h_{3}(x, y, z)\right)
$$

also belongs to $\Phi$.

This shows, by the definition of $\boldsymbol{A}_{0}^{(3)}$ (cf. [3], sec. 1, (a)) that $\boldsymbol{A}_{0}^{(8)}=\Phi$. Knowing $\boldsymbol{A}_{0}^{(3)}$ we easily check that every three elements are independent.

Uniqueness of $\mathscr{A}_{0}$. We now proceed to prove Theorem 4 . We assume that the algebra $\mathscr{A}=(\boldsymbol{A}, \boldsymbol{A})$ is non-trivial, independentily generated by every three elements and $\overline{\bar{A}} \geqslant 3$ (where $A$ denotes the class of all algebraic operations). We first show that

( $\gamma)$ If $f \in A^{(3)}$ is non-trivial, then identically

$$
f(x, x, y)=f(x, y, x)=f(y, x, x)=y \text {. }
$$

Proof of $(\gamma)$. Let $a, b, o$ be independent generators of $s$. If $f$ is non-trivial, we have $f(a, b, c) \neq a, b, c$ and thus $a, b, f(a, b, c)$ generate Al. It follows that, for a certain algebraic operation $h(x, y, z)$,

$$
c=h(a, b, f(a, b, c)) \text {. }
$$

Since $a, b, c$ are independent, this equation holds identically, e. g. also if $a$ stainds at the place of $b$. So $c=h(a, a, f(a, a, c))$. Now $f(x, x, y)$ is, by $(\alpha)$, a trivial operation, whence $f(x, x, y)=x$ or $y$. But $f(a, a, c)=a$ gives $0=h(a, a, a)$, which is a contradiction. Thus we have $f(x, x, y)=y$. The other equalities in $(\gamma)$ hold by symmetry.

Now let us show that $\mathscr{A}$ has exactly four elements. We have assumed $\bar{A} \geqslant 3$. Since $\mathscr{A}$ is non-trivial and every three elements are independent, we have, by Theorem $1, \overline{\bar{A}}>3$. Let us suppose that there are at least five elements $a, b, c, d, e \in A$. Since $a, b, c$ are generators, there are operations $f, g$ such that

$$
d=f(a, b, c), \quad e=g(a, b, c) .
$$

Also $c, d, e$ are generators; thus, for some operation $h, a=h(c, d, e)$
we have and we have

$$
a=h(c, f(a, b, c), g(a, b, c)) .
$$

Since this equality must hold identically, we have, writing $a$ at the place of $b$, by $(\gamma), a=h(c, c, c)$. This is a contradiction and so $\bar{A}=4$.

From what we have shown it follows that we may assume that both algebras, $\mathcal{L}_{0}$ and $\mathcal{A}$, have the same set of elements $A_{0}=A$ $=\{a, b, c, d\}$. To complete the proof of our theorem it remains to prove that the operations are in both algebras the same, i. e. that $\boldsymbol{A}_{0}=\boldsymbol{A}$.
Since $a, b, c$ generate $\varsigma$, we have, for a certain $f \in A^{(3)}, d=f(a, b, c)$. Thus $f$ is non-trivial and hence it associates with every three elements of $A$ the remaining one. Since the equalities in $(\gamma)$ hold, we infer that $f$ coincides with the fundamental operation $f_{0}$ of $s \tau_{0}$. Consequently $\boldsymbol{A}_{0}$ is the class of operations generated by $f$ and we have $\boldsymbol{A}_{0} \subset \boldsymbol{A}$.

Given an operation $h \in \boldsymbol{A}^{(k)}$, we say that $h$ depends on the variable $x_{i}$, where $1 \leqslant i \leqslant k$, if there is a sequence $\left\langle a_{1}, \ldots, a_{i}, \ldots, a_{k}\right\rangle$ of elements of $A$ and an $a_{i}^{\prime} \in A$ such that

$$
h\left(a_{1}, \ldots, a_{i}, \ldots, a_{k}\right) \neq h\left(a_{1}, \ldots, a_{i}^{\prime}, \ldots, a_{k}\right) .
$$

We have to show that $\boldsymbol{A} \subset \boldsymbol{A}_{0}$. We observe that if $h \in A$, then $h$ must depend on some variables, for if $h$ takes a constant value, say $h=a$, then also $h(b, \ldots, b)=a$, contradicting the independence of $a$ and $b$. We can even assume that $h$ depends on every variable, for if $h\left(x_{1}, \ldots, x_{k}\right)$ does not depend on some of the variables, then, after a suitable rearrangement of indices if necessary, we have

$$
h\left(x_{1}, \ldots, x_{k}\right)=g\left(x_{1}, \ldots, x_{m}\right), \quad m<k ;
$$

$g$ depends on every variable and if $g \in A_{0}$, then $h \in A_{0}$.

The idea of our proof is now the following. To show that if $h \in A^{(k)}$ depends on every variable, then $h \in A_{0}^{(k)}$, it is enough to verify that there is at most one operation in $A^{(l)}$ which depends on every variable, and, if there is one, then there is at least one which belongs to $A_{0}^{(k)}$. This follows, by $\boldsymbol{A}_{0} \subset \boldsymbol{A}$, from.

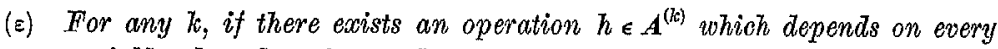
variable, then there is exactly one such operation and $k$ is an odd integer.

( $)$ For every odd integer $k$, there exists an operation $h \in A_{0}^{(k)}$ which depends on every variable.

Since the proof of $(\eta)$ is much simpler than the proof of $(\varepsilon)$, we shall give it first.

Proof of $(\eta)$. The assertion is trivial for $k=1$. Suppose that, for some odd $k \geqslant 1$, there is an operation $h\left(x_{1}, \ldots, x_{k}\right)$ belonging to $\boldsymbol{A}_{0}^{\left(k_{k}\right)}$ and depending on every variable. Then the operation $g$ defined by

$$
g\left(x_{1}, \ldots, x_{k+12}\right)=f\left(h\left(x_{1}, \ldots, x_{k}\right), x_{k+1}, x_{k+2}\right)
$$

belongs to $\boldsymbol{A}_{0}^{(k+2)}$. Trrom $g\left(x_{1}, \ldots, x_{k}, x, x\right)=h\left(x_{1}, \ldots, x_{k}\right)$ we infer that $g$ depends on each of the variables $x_{1}, \ldots, x_{k}$. Since, for a constant $u \in A$, the function $f(u, x, y)$ depends on each of the variables $x, y$, it follows that $g$ depends also on $x_{k+1}$ and $x_{k+2}$.

Proof of $(\varepsilon)$. Since, by $(a)$, every operation of not more than two variables is trivial, ( $\varepsilon$ ) holds for $k=1$ and $k=2$. Let us show that $(\varepsilon)$ holds for $k=3$, i. e., that $f(x, y, z)$ is the only operation in $A^{(3)}$. which depends 
on all variables. Suppose that $g(x, y, z) \in \boldsymbol{A}^{(3)}$ depends on every variable. Hence $g$ is a non-trivial operation and we must have $g(a, b, 0)=d$, by the independence of $a, b, c$. So $g(a, b, c)=f(a, b, a)$ and, using again the independence of $a, b, c, g(x, y, z)=f(x, y, z)$.

Thus it remains to prove $(\varepsilon)$ for $k>3$. Suppose that $h \in \boldsymbol{A}^{(k)}, k>3$ and $h$ depends on every variable. Since we have only four elements in $A$, the operation $h$ is given by a system of operations of not more than four variables which are obtained from $h$ by identifying some of the variables $x_{1}, \ldots, x_{k}$ so that not more than four different ones are left. Let us call those operations derived from $h$. If we show that each operation derived from $h$ depends only on the given identification of the variables and not on $h$, then it is evident that $h$ is nnique. This proof will be given in two steps. First, in $\left(\varepsilon_{0}\right)$, we show that each operation $h_{\delta}$ derived from $h$ is determined by the system of operations of two variables derived from $h_{8}$. Then, in $\left(\varepsilon_{1}\right)$, we prove that the system of operations of two variables derived from $h$ can be determined without knowing $h$.

We consider the family of all decompositions of the set of indices $S=\{1, \ldots, k\}$ in not more than four subsets. For each decomposition $\delta$

$$
S=X \cup Y \cup Z \cup U
$$

(where some of the sets $X, \ldots, U$ may be empty) we identify those variables in $h\left(x_{1}, \ldots, x_{k}\right)$ which have indices belonging to the same set of the decomposition. We denote by $x, y, z, u$ those variables $x_{i}$ whose indices belong to the sets $X, \ldots, U$ respectively. Thus we obtain an operation $h_{\delta}(x, y, z, u)$, which is derived from $h$.

$\left(\varepsilon_{0}\right)$ The operation $h_{\delta}(x, y, z, u)$ is determined by the system of operations of two variables whioh are derived from $h_{s}$.

Proof of $\left(\varepsilon_{0}\right)$. Our assertion is trivial if $\delta$ is a decomposition of $S$ in two sets. If $\delta$ is a decomposition in three sets, then $h_{\delta}=h_{q}(x, y, z)$. If $h_{\delta}$ is not trivial, then $h_{\delta}=f$ since $f$ is the only non-trivial algebraic operation of three variables. Consequently $h_{\delta}(x, x, y)=h_{\delta}(x, y, x)$ $=h_{\delta}(y, x, x)=y$. If $h_{\delta}$ is trivial, then only two of these equalities holds and the remaining four are false. Moreover, we know $h_{\delta}$ if we know which of the equalities holds. Thus all the possible cases are distinguished by the behaviour of the operations of two variables derived from $h_{d}$; we can determine $h_{o}$ by examining those operations.

Now suppose that $\delta$ is a decomposition of $S$ in four sets. We have to consider an operation $h_{\delta}(x, y, z, u)$. Let us determine first $h_{s}(a, b, c, d)$. Suppose that $h_{\delta}(a, b, c, d)=d$. Then $h_{\delta}(a, b, c, f(a, b, c))=f(a, b, c)$ and, by the independence of $a, b, c$, we have $h_{\delta}(x, y, z, f(x, y, z))=f(x, y, z)$. Identifying any two of the vaxiables $x, y, z$ we easily obtain

$$
h_{\delta}(x, x, y, y)=h_{\delta}(x, y, x, y)=h_{\delta}(y, x, x, y)=y \text {. }
$$

Let us call an identification of some of the variables $x, y, z, u$ even if it results in one of those which appear in brackets in the above equalities. Thus we infer that for any even identification of variables, $h_{\delta}(x, y, z, u)=u$. By symmetry, if we assume that $h_{\delta}(a, b, c, d)=c$, we shall find that, for every even identification of variables, $h_{\delta}(p, y, z, u)=z$ holds and it is similar for $a$ and $b$ instead of $c, d$. So we see that by examining the operations of two variables derived from $h_{\delta}$ (in fact only those which are derived by an even identification of variables) we can determine $h_{\delta}(a, b, c, d)$.

It remains to verify that also in the case when two of the arguments $x, y, z, u$ are equal, $h_{\delta}$ can be determined. It is not difficult to see that we then have to find the value of an operation $h_{\delta}$ of not more than three variables which is derived from $h_{b}$. Since we know the operation of two variables derived from $h_{\delta}$ (they are also derived from $h_{\delta}$ ) we determine $h_{\delta}^{-}$so in the same way as we determined $h_{\delta}$ when it was assumed to be an operation of not more than three variables. Thus $\left(\varepsilon_{0}\right)$ is proved.

Let $\Omega_{h}$ be the family of subsets of $S$ which contains, for every decomposition $\delta: S=X \cup Y$, one of the two sets $X, Y$, namely

$$
X \text { if } h_{s}(x, y)=x, \quad Y \text { if } h_{s}(x, y)=y .
$$

(Let us recall that in $\mathscr{A}$ every algebraic operation of two variables is trivial.)

Obviously

(o) $S \in \Omega_{h}$.

(i) If $G \cup H=S$ and $G \cap H=\emptyset$, then exactly one of the sets $G$ and $H$ belongs to $\Omega_{h}$.

It is evident that $\Omega_{h}$ determines the system of operations of two variables derived from $h$. Thus, by $\left(\varepsilon_{0}\right), h$ is completely determined by $\Omega_{h}$. To show that $h$ is unique it is enough to show that $\Omega_{h}$ is unique, i. e. that $\Omega_{h}$ is fully determined by the mere condition that $h$ is operation of $k$ variables which depends on every variable. This will be shown in $\left(\varepsilon_{1}\right)$, but to prove $\left(\varepsilon_{1}\right)$ we need some more properties of $\Omega_{h}$. Assume that $\theta, H \in \Omega_{h}$, Let us prove

(ii) If $G \cap H \neq \emptyset$ and $S=G \cup H$, then $G \cap H \in \Omega_{h}$.

(iii) If $G \cap H=\emptyset$, then $G \cup H \notin \Omega_{h}$.

(iv) If $G \subset H$, then $H-G \notin \Omega_{h}$.

In the proofs of (ii), (iii) and (iv) we shall consider an operation $h_{0}(x, y, z)$ given by a decomposition $\delta: S=X \cup Y \cup Z$. The sets $X, Y, Z$ will be defined in each case separately.

Proof of (ii). Assume $G \cap H \neq \emptyset, S=G \cup H$. Define $X=\theta-H$, $Y=G \cap H, Z=H-G$. Consider the operation $h_{\delta}(x, y, z)$. We have $X \cup Y, Z \cup Y \in \Omega_{h}$ and thus $h_{b}(x, x, y)=x$ and $h_{b}(x, y, y)=y$. Hence 
$h_{b}$ is different from $f$ and thus it is a trivial operation. Consequently $h_{\delta}(x, y, z)=y$. It follows that $h_{\delta}(x, y, x)=y$ and thus (ii).

Proof of (iii). Suppose that $G \cap H=\emptyset$. Let $X=G, Y=H$, $Z=S-(G \cup H)$. From $X, X \in \Omega_{h}$ it follows $h_{\delta}(x, y, y)=x, h_{\delta}(x, y, x)=y$ and thus $h_{\delta}$ cannot be a trivial operation. Hence $h_{\delta}(x, y, z)=f(x, y, z)$ and we have $h_{\delta}(x, x, z)=z$. Thus $Z \in \Omega_{h}$ and consequently $G \cup H \notin \Omega_{h}$.

Proof of (iv). Let $G \subset H$. Define $X=G, X=S-H, Z=H-G$. It follows from $X, X \cup Z \in \Omega_{h}$ that $h_{s}(x, y, y)=x=h_{\delta}(x, y, x)$. We see that $h_{\delta}$ is different from $f$. Thus it is a trivial operation satisfying $h_{s}(x, y, z)=x$. For $x=y$ we obtain $Z \notin \Omega_{h}$. This proves (iv).

We shall derive from $\left(\varepsilon_{0}\right)$, (ii) and (iii) that

(v) Every one-element subset of $S$ belongs to $\Omega_{n}$.

Pro of of (v). Without loss of generality it will be enough to prove that $\{1\} \in \Omega_{h}$. Since $h\left(x_{1}, \ldots, x_{\delta}\right)$ depends on $x_{1}$, there are sequences $\left\langle a_{1}, \ldots, a_{k}\right\rangle,\left\langle b_{1}, \ldots, b_{k}\right\rangle, a_{i}, b_{i} \in A$ such that $a_{i \omega}=b_{i}$ for $i \geqslant 2$ and

$$
h\left(a_{1}, \ldots, a_{k}\right) \neq h\left(b_{1}, \ldots, b_{k}\right) .
$$

Since obviously $a_{1} \neq b_{1}$, we can assume that $a_{1}=a, b_{1}=b$. Both sequences, $\left\langle a_{1}, \ldots, a_{k}\right\rangle$, and $\left\langle b_{1}, \ldots, b_{k}\right\rangle$, are composed of the elements $a, b, c, d$, and thus there are uniquely determined decompositions is and $\bar{\delta}$ of $S$.

such that

$$
B=X \cup Y \cup Z \cup U, \quad S=\bar{X} \cup \bar{Y} \cup \bar{Z} \cup \bar{U}
$$

$$
h\left(a_{1}, \ldots, a_{k}\right)=h_{\delta}(a, b, c, d) ; \quad h\left(b_{1}, \ldots, b_{k}\right)=h_{\delta}(a, b, c, d) .
$$

It is not difficult to check that $\bar{X}=\bar{X}-\{1\}, \bar{Y}=\bar{X} \cup\{1\}, \bar{Z}=Z$, $\bar{U}=U$. We see also that the operations $h_{\delta}$ and $h_{\bar{\delta}}$ are different.

Let $O_{1}, O_{2}, C_{3}, C_{4}$ stand for the symbols $X, Y, Z, U$ but not necessarily in the same order. We find, by $h_{\delta} \neq h_{\delta}$ and by $\left(\varepsilon_{0}\right)$ that there are two different operations of two variables, one derived from $h_{\delta}(x, y, z, u)$, the other from $h_{\bar{\delta}}(x, y, z, u)$ and both obtained by the same identification of some of the variables $x, y, z, u$. This means that there is a set $C \subset S$ which can be denoted by $O_{1} \cup C_{2} \cup C_{3}$ or by $C_{1} \cup C_{2}$ or by $C_{1}$ such that $C \in \Omega_{h}$ but the corresponding set $\bar{C} \subset \bar{S}$ denoted by $\bar{C}_{1} \cup \bar{C}_{2} \cup \bar{C}_{3}$ or $\bar{O}_{1} \cup \bar{O}_{2}$
or $\bar{O}_{1}$ does not belong to $\Omega_{h}$.

It is obvious that $\bar{O}$ and $\bar{C}$ differ only in the element 1 of $S$ and we have either $\{1\}=C-\bar{C}$ and $\bar{C} \subset O$ or $\{1\}=\bar{C}-C$ and $O C \bar{C}$. We define $G=C, H=S-\bar{C}$ so that $G \in \Omega_{h}$ and, by (i), $H \in \Omega_{h}$.

If $\{1\}=O-\bar{C}$, then $S=G \cup H$ and $G \cap H=\{1\}$. We derive from (ii) that $\{1\} \in \Omega_{h}$. If $\{1\}=\bar{C}-O$, we have $Q \cap H=\varnothing$ and therefore, by (iii), $G \cup H \notin \Omega_{h}$ and, by (i), $\{1\} \in \Omega_{h}$.
We are now in a position to prove that $\Omega_{h}$ does not depend on $h$. By applying induction on the number of elements in $X$ and using (o), (i), (iii), (iv) and ( $v$ ) we find that

( $\left.\varepsilon_{1}\right)$ A set $X \subset S$ belongs to $\Omega_{h}$ if and only if the number of elements in $X$ is odd. In particular, since $S \in \Omega_{h}, k$ is odd.

As we noticed above, $\left(\varepsilon_{1}\right)$ implies that there is at most one operation $h \in \boldsymbol{A}^{(k)}$ which depends on every variable. Since $\gamma$ must then be odd, our proof of $(\varepsilon)$ and of the theorem is now complete.

\section{Algebras independently generated by every two eloments}

We shall prove here Theorems 5 and 6 . Consider first the algebra st defined in Theorem 5 . It is easy to see that the class of all algebraic operations $\boldsymbol{A}$ consists of operations of the form

$$
f\left(x_{1}, \ldots, x_{k}\right)=\lambda_{1} x_{1}+\ldots+\lambda_{k} x_{k}
$$

where $\sum_{i} \lambda_{i}=1$. Let $a, b$ be distinct elements in $A$. Then there exists, for every $c \in A$, exactly one operation $f \in A^{(2)}$ for which $c=f(a, b)$ (since the equations $\lambda_{1} a+\lambda_{2} b=c, \lambda_{1}+\lambda_{2}=1$ determine $\lambda_{1}$ and $\lambda_{2}$ ). Suppose that $g(a, b)=h(a, b)$ holds for some $g, h \in A^{(2)}$. It follows that $g=h$ and thus $a, b$ are independent. Obviously these two elements generate the algebra and thus we have proved Theorem 5 .

To prove Theorem 6 suppose that $s t$ is an algebra which is independently generated by every two elements. A group $T$ of one-to-one mappings of a set onto itself is called doubly transitive when it contains one or more mappings changing given two elements $a, b$ into any two elements $c, d$. If the conditions $c=t(a), d=t(b)$ determine uniquely the mapping $t \in T$, then $T$ is said to be minimal. We prove first that

( $\lambda$ ) The group $T$ of all automorphisms of $s t$ is a doubly transitive and minimal group of one-to-one mappings of $A$ onto itself.

Proof of ( $\lambda$ ). If $a, b$ are distinct elements of $A$ and $c, d$ also, then, by the independence of $a, b$, we infer that the mapping $a \rightarrow c, b \rightarrow d$ has an extension to a homomorphism $t$ of the subalgebra generated by $a, b$ on the subalgebra generated by $c, d$ and obviously this extension is unique (cf. [3], sec. 2, (ii)). Since $a, b$ generate $s, t$ is an endomorphism and since $c, d$ generate $\mathcal{A}, t$ is onto. Finally, from the independence of $c, d$ it follows that $t$ is an automorphism. Hence $T$ is doubly transitive and since $t$ is determined uniquely by the conditions $t(a)=c, t(b)=d, T$ is minimal.

Our theorem now follows by $(\lambda)$ since it is well known that if there exists, for a finite set, a doubly transitive and minimal group of oneto-one mappings of this set onto itself, then the number of elements of this set is a power of a prime (cf. [1], see. 105; also [2]). 


\section{References}

1] W. Burnside, Theory of groups of finite order, Cambridge 1897.

[2] - On donbly transitive groups of degree $n$ and order $n(n-1)$, The Messenger of Mathematics XXV (1896), p. 147.153.

[3] E. Marozew ski, $A$ general schene of the notions of independence in mathematics, Bull. Acad. Pol. Sci. Série math., astr. et phys. 6 (1958), p. 731.736.

4] S. Swierczkowski, Algebras independently generated by every $n$ elements, Bull. Acad. Pol. Sci. Série math., astr. et phys. 7 (1959), p. 501-502.

[5] K. U'rbanik, A representation theorem for Marczews7ti's algebras, Fund. Math. $48(1960)$, p. $147-167$

INSTYTUT MATEMATYCZNY POLSKTET AKADEMII NAUK MATHEMATICAL INSTITUTE OF THE POLISH ACADEMY OF SCIENCES

Reģu par la Rédaction le 30. 12. 1959

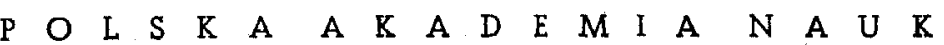

\section{FUNDAMENTA M A T H E M A I C A E}

2 A L O Z I C I E L E:

ZYGMUNT JANISZEWSKI, STEFAN MAZURKIEWICZ i WACLAW SIERPIŃSKI

KOMITET REDAKCYINY:

WACEAW SIERPIŃSKI, REDAKTOR HONOROWY, KAZ I M I ERZ K URATOW K I, REDAKTOR, K A R O I B O R S U K, ZASTEPCA REDAKTORA, BRONISEAW KNASTER, EDWARD MARCZEWSKI. STANISLAW MAZUR, ANDRZEJ MOSTOWSKI

\section{2}

